\title{
Nonadiabatic Molecular Dynamics Simulations of the Energy Transfer between Building Blocks in a Phenylene Ethynylene Dendrimer ${ }^{\dagger}$
}

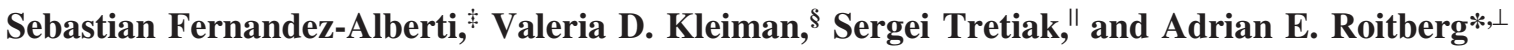 \\ Universidad Nacional de Quilmes, Roque Saenz Peña 352, B1876BXD Bernal, Argentina, Department of \\ Chemistry, University of Florida, Gainesville, Florida 32611, Theoretical Division, Center for Nonlinear \\ Studies (CNLS), and Center for Integrated Nanotechnologies (CINT), Los Alamos National Laboratory, \\ Los Alamos, New Mexico 87545, and Quantum Theory Project, Department of Chemistry, \\ University of Florida, Gainesville, Florida 32611
}

Received: January 30, 2009; Revised Manuscript Received: March 20, 2009

The ultrafast dynamics of electronic and vibrational energy transfer between two- and three-ring linear poly(phenylene ethynylene) units linked by meta-substitution is studied by nonadiabatic molecular dynamics simulations. The molecular dynamics with quantum transitions ${ }^{1,2}$ method is used including an "on the fly" calculation of the potential energy surfaces and electronic couplings. The results show that during the first 40 fs after a vertical photoexcitation to the $S_{2}$ state, the nonadiabatic coupling between $S_{2}$ and $S_{1}$ states causes a fast transfer of the electronic populations. A rapid decrease of the $S_{1}-S_{2}$ energy gap is observed, reaching a first conical intersection at $\approx 5$ fs. Therefore, the first hopping events take place, and the $S_{2}$ state starts to depopulate. The analysis of the structural and energetic properties of the molecule during the jumps reveals the main role that the ethynylene triple bond plays in the unidirectional energy transfer process.

\section{Introduction}

The fundamental principles of the efficient energy funneling observed in light-harvesting photosynthetic systems can be unraveled by studying the built-in energy gradients in welldefined, artificial light-harvesting macromolecules called dendrimers. Such dendrimers are highly branched conjugated macromolecules with regular structures possessing numerous peripheral groups, branched repeat units, and a core. Their unique architecture allows an efficient and controllable vectorial energy transport from the periphery to the core. In most cases, the branched repeat units act as selected photoactive chemical units that constitute a suitable media for the exciton transport and relaxation during the light-harvesting processes. Therefore, the detailed understanding of the unidirectional photoexcitation energy transfer between the repeat units inside a dendritic macromolecule is a very important goal in the field of organic photochemistry.

Apart from their fundamental interest, the controlled synthesis of dendrimers awards them with unique geometric and energetic properties $^{3}$ suitable for a wide variety of applications, including molecular devices and machines, ${ }^{4}$ the encapsulation of small organic molecules in their "dendritic box" acting as drug delivery systems, ${ }^{5,6}$ and the enhancement of the guests' luminescence properties attributed to their large antenna effect. ${ }^{7}$ In particular, the family of dendrimers based on poly(phenylene ethynylene) (PPE) units has received a special theoretical and experimental interest due to their ability to mimic both the collection and energy transport that occur in photosynthetic systems. ${ }^{8-13}$ Since they can be prepared with high regularity

\footnotetext{
"Part of the "Robert Benny Gerber Festschrift".

* To whom correspondence should be addressed.

* Universidad Nacional de Quilmes.

$\S$ Department of Chemistry, University of Florida.

"Los Alamos National Laboratory.

${ }^{\perp}$ Quantum Theory Project, Department of Chemistry, University of Florida.
}

and controlled molecular weights, ${ }^{14}$ dendrimers offer great promise in the control of energy funneling. Their successful directional-energy transfer has been studied by Moore et al., $15,16,9,17$ who have reported the syntheses of a peryleneterminated dendrimer, called the nanostar. This molecule presents four generations of linear PPE segments with decreasing lengths toward the periphery, creating an efficient energy funnel toward the perylene trap. An important feature of the nanostar is that the different PPE segments are linked by metasubstitutions at the branching phenylene nodes. These connecting nodes inhibit any conjugate delocalization of electrons across the dendrimer framework. Therefore, the dendrimer molecule can be understood as an ensemble of linear chromophore units with weak coupling between them. As a result, the excitons in the nanostar are localized on each PPE segment, ${ }^{9}$ and its overall absorption spectrum can be interpreted as the sum of these individual contributions. ${ }^{15,10,17}$

Theoretical interest in PPE dendrimers arises from the possibility of analyzing them as an ensemble of individual coupled chromophore units. Therefore, the system can be described using the Frenkel exciton Hamiltonian ${ }^{18,19}$ with Coulombic coupling between excitations localized on the linear units. ${ }^{20}$ Its combination with the collective electronic oscillator $(\mathrm{CEO})^{21,22}$ approach has been proved to be suitable for the calculation of electronic spectra in a large family of dendrimers and conjugated chromophores. ${ }^{23,47}$ The CEO model uses realspace wave packets representing the single-electron density matrix. The optical properties of the system are related directly to the motions of charges and electronic coherences. For this purpose, collective electronic normal modes or transition density matrices obtained using time-dependent Hartree-Fock (TDHF) are required. Furthermore, using this theoretical framework, Mukamel and collaborators simulated the exciton transport and relaxation in PPE dendrimers using the Redfield equations. ${ }^{24-26}$

The localization of the excitations on the different branched units in the nanostar has been previously shown using molecular 


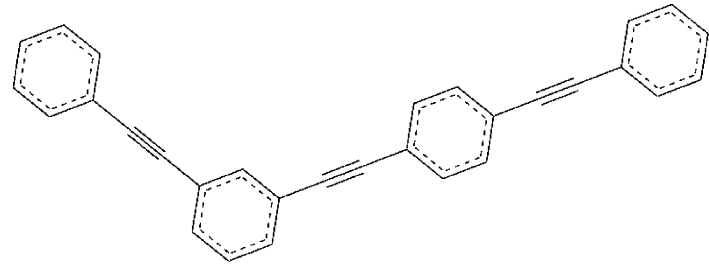

Figure 1. Molecule studied in this work. It involves two- and threering linear poly(phenylene ehynylene) units linked by meta-substitution.

dynamics and quantum mechanical calculations. ${ }^{27,28}$ These results indicate that the nanostar can be studied as the sum of separate linear PPE units: two-ring, three-ring, and four-ring para-substituted phenylene ethynylene. The three main peaks in the absorption spectrum correspond to the absorption of each one of these photoactive units. That is, the peak at shorter wavelength $(310 \mathrm{~nm})$ corresponds to the two-ring system, the next peak $(352 \mathrm{~nm})$ in ascending order of wavelength corresponds to the three-ring system, and the peak at higher wavelength $(372 \mathrm{~nm})$ corresponds to the four-ring system.

However, while all the transitions are well localized at low temperature $(\sim 10 \mathrm{~K})$, the situation becomes more complicated at higher temperatures. In a previous work, ${ }^{28}$ we have shown that the absorptions of the three-ring systems and the four-ring systems are shifted to lower wavelengths leading to the superposition of the different contributions to the absorption spectra at $300 \mathrm{~K}$. The origin of these displacements can be understood analyzing the many different possible conformations that the PPE units can adopt during the molecular dynamics simulation. The results indicated that the rings lie essentially in one plane at low temperature whereas they rotate freely at room temperature. This change in geometry was proven to have a significant effect on the transition density, and hence energy transfer rates. ${ }^{29}$ Therefore, the present work proposes to relate the vibrational motions of the PPE units with their efficiency in the unidirectional energy transfer by using direct nonadiabatic molecular dynamics simulations.$^{30}$ Previous works have already shown the usefulness of these methods in the study of photochemical reaction mechanisms in organic compounds. ${ }^{31-35}$ In particular, direct dynamics studies using trajectory surface hopping have been applied to the photochemistry of a wide variety of organic molecules: benzene, ${ }^{36}$ fulvene, azulene, ${ }^{37}$ guanine-cytocine,${ }^{38}$ formamide, ${ }^{39}$ silaethylene, ${ }^{40}$ ethylene, ${ }^{41}$ and pyrrole, ${ }^{42}$ among others. The level of accuracy on the excitedstate electronic energies, gradients, and nonadiabatic couplings varies according to specific features of the system under study.

Femtosecond degenerate pump-probe spectroscopy has been carried out by Kleiman et al. ${ }^{43}$ to study the ultrafast dynamics of electronic excitations in the nanostar dendrimer. Preferential excitation at $310 \mathrm{~nm}$, which corresponds to excitation of the two-ring unit, yields a biexponential decay ( $\sim 3$ and $\sim 15 \mathrm{ps})$ indicating multiple pathways governed by the energy funnel. Those experiments provided a measure of the time scale for energy transfer out of the initially pumped two-ring chromophores. Further experiments utilizing broad-band transient absorption and ultrafast time-resolved emission elucidate a complex picture of energy transfer through multiple channels including vectorial energy transfer through the individual phenylene ethynylene units as well as parallel channels connecting units which are not necessarily covalently coupled. ${ }^{44}$

In this work, the ultrafast dynamics of electronic and vibrational energy transfer between two-ring and three-ring PPE units separated by meta-substitution (Figure 1) is studied by nonadiabatic molecular dynamics simulations. We use molecular dynamics with quantum transitions (MDQT) based on Tully's stochastic fewest-switches algorithm. ${ }^{1,2}$ Since the process involves electronic transitions among the different electronic excited states, our aim is to stress the role of nonadiabatic couplings in the description of the energetics of the system during the ultrafast dynamics after the photoexcitacion. Structural and dynamical features were particularly examined in the region of strong nonadiabatic coupling, revealing the chemical principles for the efficient energy transfer process.

\section{Methods}

A. The MDQT Method. We have performed nonadiabatic molecular dynamics simulations of the electronic and vibrational energy transfer between the two-ring and three-ring PPE units schematized in Figure 1. The MDQT method was used as implemented by Tully. ${ }^{1,2}$ Briefly, the method treats the electronic degrees of freedom quantum mechanically, while the motion of the nuclei is treated classically. The nuclei evolve on a potential energy surface (PES) that is defined by a single electronic state at a given time. Jumps from one electronic state to another are governed by the coefficients of the electronic wave function. More details can be found in refs 1 and 2 .

In the present work the electronic wave function is written as

$$
\Psi(\mathbf{r}, \mathbf{R}, t)=\sum_{I}^{N_{\mathrm{CI}}} C_{I}(t) \psi_{I}(\mathbf{r} ; \mathbf{R})
$$

where $\mathbf{r}$ and $\mathbf{R}$ are the electronic and nuclear vector coordinates, respectively. $N_{\mathrm{CI}}$ is the number of configuration interaction (CI) states considered. $\psi_{I}(\mathbf{r} ; \mathbf{R})$ is the $\mathrm{CI}$ eigenstate defined in terms of the configuration state functions (CSFs) $\phi_{i}(\mathbf{r} ; \mathbf{R})$ as

$$
\psi_{I}(\mathbf{r} ; \mathbf{R})=\sum_{i} c_{I, i} \phi_{i}(\mathbf{r} ; \mathbf{R})
$$

The CI coefficients $c_{I, i}$ are obtained solving the matrix eigenvalue equation

$$
H(\mathbf{R}) c_{I}(\mathbf{R})=E_{I}(\mathbf{R}) c_{I}(\mathbf{R})
$$

where

$$
H_{i j}(\mathbf{R})=\left\langle\phi_{i}(\mathbf{r} ; \mathbf{R})|H(\mathbf{r} ; \mathbf{R})| \phi_{j}(\mathbf{r} ; \mathbf{R})\right\rangle_{\mathbf{r}}
$$

where $H$ is the standard nonrelativistic Hamiltonian operator. Accordingly with the configuration interaction singles (CIS) technique, the molecular orbitals (MO) of the ground state are used to construct the $\phi_{i}(\mathbf{r} ; \mathbf{R})$.

The coefficients $C_{I}(t)$ evolve in time according to

$$
i \hbar \dot{C}_{I}(t)=C_{I}(t) E_{I}-i \hbar \sum_{J} C_{J}(t) \dot{R} d_{I J}
$$

where

$$
d_{I J}=\left\langle\psi_{I}(\mathbf{r} ; \mathbf{R}) \mid \nabla_{R} \psi_{J}(\mathbf{r} ; \mathbf{R})\right\rangle
$$

is the nonadiabatic coupling vector.

The probability for a quantum transition from the current electronic state $I$ to all the other states $J$ is calculated at each integration step along the trajectory as

$$
g_{I \rightarrow J}=-\frac{\Delta t}{\hbar} \frac{2 \operatorname{Re}\left\{C_{I}(t) C_{J}^{*}(t) \dot{R} \cdot d_{I J}\right\}}{\left|C_{I}(t)\right|^{2}}
$$

with the condition that if $g_{I \rightarrow J}<0$ then $g_{I \rightarrow J}=0$. A uniform random number $0<\eta<1$ is sampled to determine whether a 
hop from the $\left|\psi_{I}(\mathbf{r} ; \mathbf{R}>)\right\rangle$ surface is realized. If the states are ordered as $I, J, K, \ldots$, then if $0<\eta \ll g_{I \rightarrow J}$, the system hops to surface $\left|\psi_{J}(\mathbf{r} ; \mathbf{R})\right\rangle$, if $g_{I \rightarrow J}<\eta<g_{I \rightarrow J}+g_{I \rightarrow K}$, the system hops to surface $\left|\psi_{K}(\mathbf{r} ; \mathbf{R})\right\rangle$ and so on, and if $\sum_{J \neq I} g_{I \rightarrow J}<\eta<1$, then the system remains in the state $I$.

If a hop $I \rightarrow J$ occurs, the trajectory continues on the new potential energy surface $E_{J}(\mathbf{R})$ with a velocity adjustment in order to conserve total energy. According to the prescription suggested by previous works, ${ }^{1,45}$ the adjustment was performed in the direction of the nonadiabatic coupling vector.

B. Potential Energy Surfaces and Couplings. The groundstate and 10 excited-state electronic energies, gradients, and nonadiabatic coupling vectors were calculated at the configuration interaction singles (CIS) level using the collective electronic oscillator (CEO) $\operatorname{code}^{22}$ combined with the Austin model 1 (AM1) Hamiltonian. ${ }^{46}$ These methodologies have been previously successfully applied to describe conjugated polymers, ${ }^{21,47}$ carbon nanotubes ${ }^{48}$ and other organic molecular materials. ${ }^{23}$ AM1 and similar semiempirical models yield reasonable estimates of vertical excitation energies, polarizabilities, and excited-state adiabatic surfaces (for comparisons with experimental data and ab initio simulations see, e.g., refs 49-52 and 46. The AM1 allows the treatments of gradients and vibrations at a significantly reduced computational cost compared to ab initio calculations. ${ }^{53}$ The CEO code computes analytic gradients of the excited-state potential energy surfaces required during the molecular dynamics simulations. ${ }^{51,52}$ Details about the method can be found elsewhere. ${ }^{23,51,52}$

The nonadiabatic couplings $\dot{R} d_{I J}$ in eq 5 have been computed numerically using finite differences to evaluate the derivative of the wave function with respect to time. Hence, the nonadiabatic coupling vector $d_{I J}$ is not required explicitly to obtain the time evolution of the CI coefficients (eq 5). Therefore, $d_{I J}$ is only calculated for the adjustment of nuclear momenta at the time of hops. For this purpose, a numerical procedure has been used. ${ }^{54-56}$

C. Molecular Dynamics Simulations. Nonadiabatic molecular dynamics simulations of the photoexcitation and energy transfer between meta-branched PPE units, shown in Figure 1, were performed using the Langevin equation at constant temperature. ${ }^{57}$ A friction coefficient $\gamma$ of $2.0 \mathrm{ps}^{-1}$ was used. This value has been chosen to allow an efficient temperature coupling. 57

First, a molecular dynamics simulation of 300 ps was run in the ground state. The system was heated and allowed to equilibrate during the first $100 \mathrm{ps}$ arriving at a final temperature of $300 \mathrm{~K}$. The last 200 ps were used to collect a set of initial positions and momenta for the subsequent simulations in the excited states.

Nonadiabatic trajectories were started from these initial configurations after a vertical excitation to the $S_{2}$ state. According to previous fragment molecular orbital analysis ${ }^{27}$ performed on TDDFT calculations at the B3LYP level with the $6-31 G(d, p)$ basis set, the $S_{2}$ electronic state is localized on the two-ring unit while the $S_{1}$ is also localized on the three-ring. The vertical excitation energies for the $S_{1}$ and $S_{2}$ states calculated at the AM1/CIS level for an AM1-optimized structure are compared in Table 1 with TDDFT (B3LYP/6-31G*) and experimental results. ${ }^{43,44}$ The agreement between the AM1 and the TDDFT results is quite good.

Each simulation was run for $40 \mathrm{fs}$ at constant temperature of $300 \mathrm{~K}$ using the MDQT method. The nuclei are propagated with the velocity Verlet integration method ${ }^{58}$ using a time step $\Delta t=$ $0.5 \mathrm{fs}$ for molecular dynamics simulations in the ground state
TABLE 1: Comparison of Experimental and Calculated Vertical Excitation Energies for $S_{1}$ and $S_{2}$ States

\begin{tabular}{lcc}
\hline \multirow{2}{*}{ method } & \multicolumn{2}{c}{$E_{\text {exc }}(\mathrm{eV})$} \\
\cline { 2 - 3 } & $\mathrm{S}_{1}$ & $\mathrm{~S}_{2}$ \\
\hline AM1/CIS & 3.24 & 3.56 \\
experiment & 3.52 & 4.0 \\
TDDFT (B3LYP/6-31G*, $10 \times 10$ CIS $)$ & 3.42 & 3.64
\end{tabular}

and a shorter time step $\Delta t=0.05$ fs for nonadiabatic molecular dynamics in the excited states. The propagation of the electronic CI coefficients (eq 5) was performed with the gear predictor corrector algorithm ${ }^{59,60}$ using a time step $\delta t 4000 \times$ shorter than the corresponding $\Delta t$ to the nuclear propagation.

The electronic energies, gradients, and nonadiabatic coupling vectors were calculated on the fly during the molecular dynamics simulations. Since we used a smaller time step for the integration of eq $5(\Delta t=4000 \delta t)$, values of energies, gradients, and nonadiabatic couplings were required at many intermediate times between $t$ and $t+\Delta t$. These values were calculated only at 10 equally spaced intermediate times, and we used a simple linear interpolation scheme $\mathrm{e}^{2}$ to obtain the values at other intermediate times.

\section{Results and Discussion}

We have carried out 200 nonadiabatic trajectories using Langevin equations at $300 \mathrm{~K}$ starting by vertical photoexcitation to the second singlet excited $\mathrm{S}_{2}$ state. These trajectories were started from the set of initial positions and momenta collected at 1.0 ps intervals from 200 ps of an equilibrated ground-state dynamics at $300 \mathrm{~K}$. At $t=0$ the system is instantaneously photoexcited to the $S_{2}$ state, while preserving the nuclear velocity distribution. Each trajectory further runs in the $S_{2}$ state until nonadiabatic couplings and energetics allow switching it to another electronic state according to the Tully's recipe described in section II.A. Figure 2 represents the average populations of the different electronic states as a function of time up to $40 \mathrm{fs}$. The results are converged with respect to the number of trajectories. An ultrafast transfer of the electronic population can be seen from the initially populated $S_{2}$ state to the closest lower singlet excited state $S_{1}$.

The fitting of the data for the population of the $S_{2}$ state based on the sum of two exponential components gives a fast component with a time constant of $3.2 \mathrm{fs}$ and a slower component that decays with a time constant of $63.0 \mathrm{fs}$. As shown

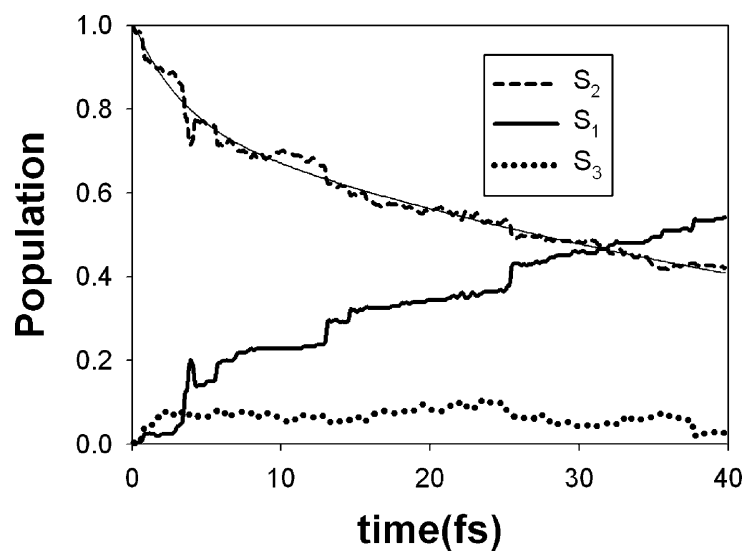

Figure 2. Population of the different electronic surfaces as a function of time obtained as the fraction of trajectories in each state. The biexponential fit for the population of the $S_{2}$ is also shown. 


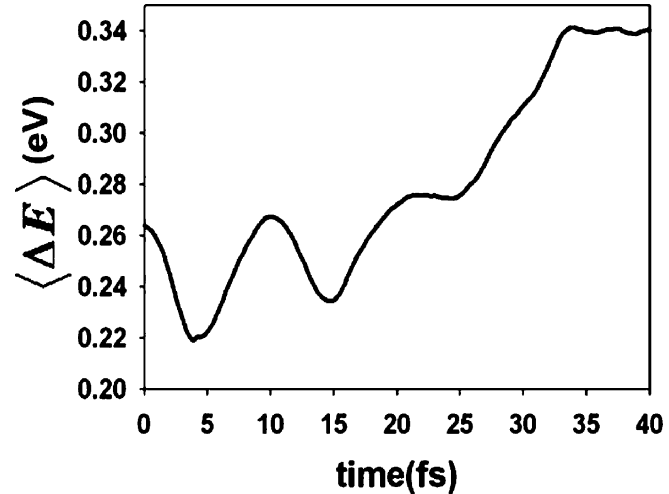

Figure 3. Time dependence of the energy gap $\Delta E$ between the $S_{2}$ and the $S_{1}$ singlet excited states averaged over all trajectories.

in Figure 2, the biexponential fit reproduces the data point very well with a correlation coefficient $R=0.99$.

These values are significantly shorter than the corresponding values reported by Kleiman et al. ${ }^{43}$ On one hand, the time scale of our simulations depends on the friction coefficient $\gamma$ used during the stochastic dynamics with the Langevin equation. Selections of larger values of $\gamma$ have been shown to decrease barrier crossing rates for conformational changes. ${ }^{61,62}$ Although this was not the focus of this study, previous works have applied Langevin dynamics to extend simulation time scales. ${ }^{63,64}$ On the other hand, the experimental measurements have been performed on the nanostar dendrimer, where the effects of the entropy and the population and coherence participation ratio should be taken into account during the exciton propagation. Particularly, the coherence effects have been shown to contribute to the transients at early times. ${ }^{43,24}$

All our exponential fits satisfy the boundary condition that all molecules must be in the $\mathrm{S}_{2}$ state at $t=0$ and they must finish in the $S_{1}$ state at the end of the process. Therefore, our results verify a highly efficient electronic energy transfer between the PPE units. This is in agreement with the near unity values of quantum yield for energy transfer to the perylene core that has been observed in the nanostar. ${ }^{15,10}$

At this point it is interesting to notice that not only the $S_{1}$ state but also the $S_{3}$ is populated during our simulations. However, its population stays below $10 \%$ mainly because the system has insufficient thermal energy to move uphill from the $S_{2}$ state. Furthermore, despite the fact that 10 CI states were taken into account during the propagation of the electronic wave function, only the $S_{3}$ shows a significant population during the dynamics. This state is close in energy to the $S_{2}$ state, and it exhibits a strong coupling with it. Nevertheless, we do not find that it plays a significant role in the energy transfer process under study, and its population starts to decrease at $\sim 25$ fs after photoexcitation.

The reason behind the very fast $S_{2} \rightarrow S_{1}$ decay can be found in the analysis of the time evolution of the energy gap $\Delta E$ between these states. On the basis of the well-known energy gap dependence of the nonradiative decay rate constants, ${ }^{65-67}$ it is expected that trajectories that cross through regions with a small energy gap will undergo large nonadiabatic couplings. In Figure 3 we present the average $\Delta E$ between the $S_{2}$ and the $S_{1}$ singlet excited states over all trajectories. It is seen that a first minimum is achieved in less than $5 \mathrm{fs}$ after the photoexcitation. Taking into account that the $\Delta E$ values calculated at the AM1 and the TDDFT(B3LYP/6-31G*) levels are 0.32 and $0.22 \mathrm{eV}$, respectively (Table 1), this first minimum seems not to be a consequence of an underestimation of the $\Delta E$ value at the AM1

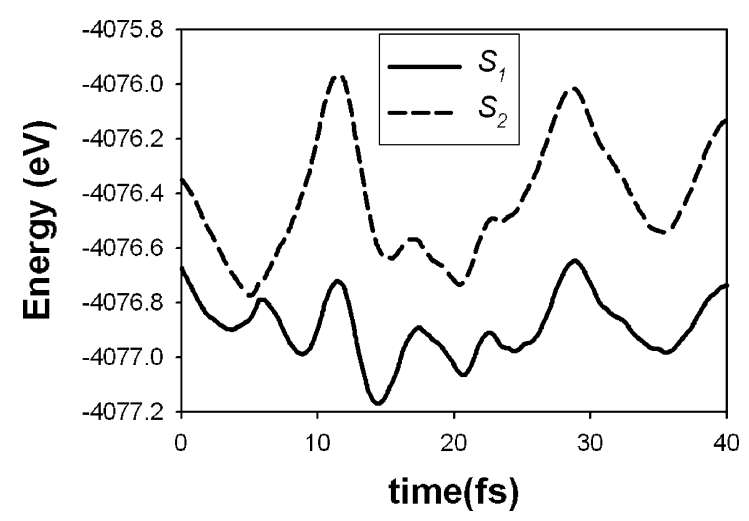

Figure 4. Time evolution of the adiabatic energies for the $S_{1}$ and $S_{2}$ states for a typical nonadiabatic trajectory.

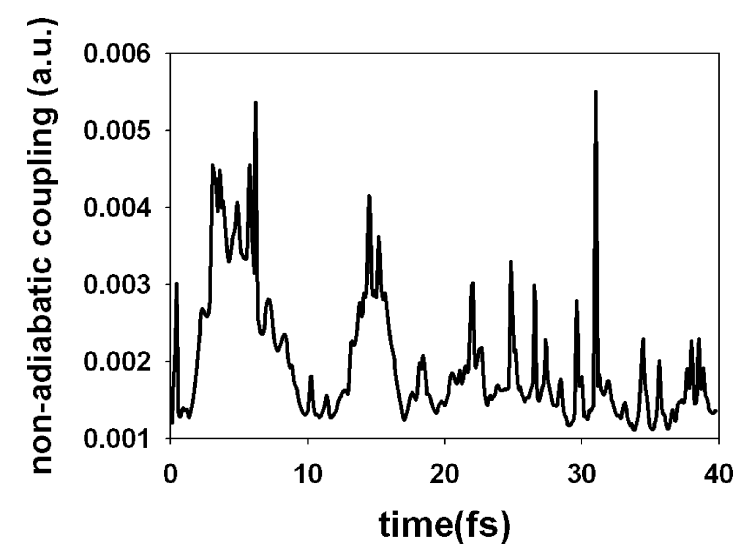

Figure 5. Time dependence of the nonadiabatic coupling $\dot{R} d_{1,2}$ averaged over all trajectories.

method. The analysis of individual trajectories reveals that some of them pass through a region of strong nonadiabatic coupling at these very early times. The time evolution of the adiabatic energies for the $S_{1}$ and $S_{2}$ states for a typical trajectory is shown in Figure 4. These trajectories present significant crossing probabilities at those times, and they mainly hop from the $S_{2}$ to the $S_{1}$ state in less than $5 \mathrm{fs}$. The set of trajectories that remains in the $S_{2}$ undergoes a second, but less marked, region of large nonadiabatic coupling and small energy gap at $\approx 15 \mathrm{fs}$. Once the system moves on the $\mathrm{S}_{1}$ state, the $\Delta E$ increases and the probability that a back-hopping (that is, $\mathrm{S}_{1} \rightarrow \mathrm{S}_{2}$ hopping) take places becomes negligible.

The time dependence of the nonadiabatic coupling $\dot{R} d_{1,2}$ averaged over all trajectories is displayed in Figure 5 . Note the two regions of large nonadiabatic coupling at $\approx 5$ and $15 \mathrm{fs}$, in agreement with regions of small $\Delta E$ (Figure 4). Thereafter, the divergence of independent trajectories, partially due to their different $S_{1} \rightarrow S_{2}$ hopping times, makes them follow different pathways on either the $S_{1}$ or $S_{2}$ potential energy surfaces. Once the system hops to the $S_{1}$ state, it moves to regions of low nonadiabatic coupling assuring the whole $S_{2} \rightarrow S_{1}$ electronic population transfer. This can be seen by inspection of Figure 6 where the average of the nonadiabatic coupling is depicted for trajectories that hop at times earlier than 10 fs (Figure 6a), earlier than $20 \mathrm{fs}(6 \mathrm{~b})$, and after $20 \mathrm{fs}(6 \mathrm{c})$. It is clearly seen from Figure 6a that trajectories that hop within the first region of large nonadiabatic coupling $(t \approx 5$ fs) move away toward regions of the configuration space with small nonadiabatic coupling. This is not the case for trajectories that remain in the $\mathrm{S}_{2}$ state ( $6 \mathrm{~b}$ and $\left.\mathrm{c}\right)$. Therefore, the difference in the nuclear dynamics on the $S_{2}$ and on the $S_{1}$ state enhances the funneling signature of the energy transfer mechanism in this molecule. 


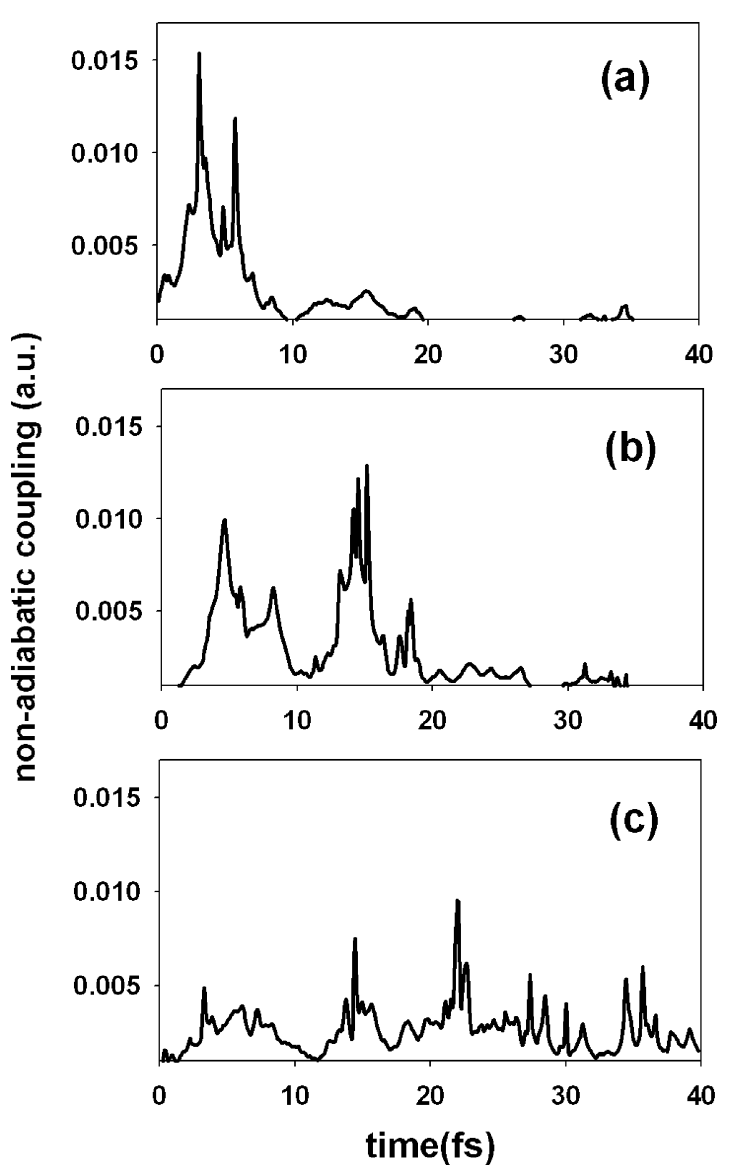

Figure 6. Time dependence of the nonadiabatic coupling $\dot{R} d_{1,2}$ averaged over all trajectories that hop at times earlier than $10 \mathrm{fs}$ (a), earlier than $20 \mathrm{fs}$ (b), and after $20 \mathrm{fs}$ (c).

We define a hop as an effective $S_{2} \rightarrow S_{1}$ transition if no backhopping occurs along the rest of the trajectory. The distribution of the number of these effective $S_{2} \rightarrow S_{1}$ hops as function of time is shown in Figure 7a. These hops follow the changes in the CI coefficients (eq 5) given by the nonadiabatic coupling displayed in Figure 5. A recurrence pattern is observed with a period of $\approx 10 \mathrm{fs}$. This pattern correlates with the minima of $\Delta E$ and the maxima of $\dot{R} d_{1,2}$ shown in Figures 3 and 5, respectively. Moreover, Figure $7 \mathrm{~b}$ presents the distribution of effective $S_{2} \rightarrow S_{1}$ hops in terms of transition energy. The average $\Delta E$ at hops events is $0.084 \mathrm{eV}$. In order to clarify whether a conical intersection (with $\Delta E=0$ ) can be reached by our trajectories, we adopt the previously proposed arbitrary limit of $\Delta E<2 \mathrm{kcal} / \mathrm{mol}=0.086 \mathrm{eV}$ to separate cases of strict conical intersections or their immediate regions and cases with larger $\Delta E$ 's. With this separation in mind, we can say that more than $60 \%$ of trajectories pass very close to a conical intersection.

Previous works have revealed that coherences between electronic excitonic states can have a substantial impact on excitation energy transfer in light-harvesting systems. ${ }^{68,69}$ For instance, it has been shown that the energy transfer in photosynthetic complexes is more suitable to be described by wavelike coherent motion instead of incoherent hopping. ${ }^{70}$ The recurrences shown in Figures 3 and 5 seem not to be related to coherences between electronic excited states, and no recurrences are observed in the time evolution of the electronic populations (Figure 2). Nevertheless, the treatment of coherence may not be optimal in the MDQT method, ${ }^{71-73}$ and the use of Langevin equations can produce a substantial effect by carefully selecting $\gamma$.

In order to analyze the structural and dynamical features that contribute to the ultrafast energy transfer, the nonadiabatic
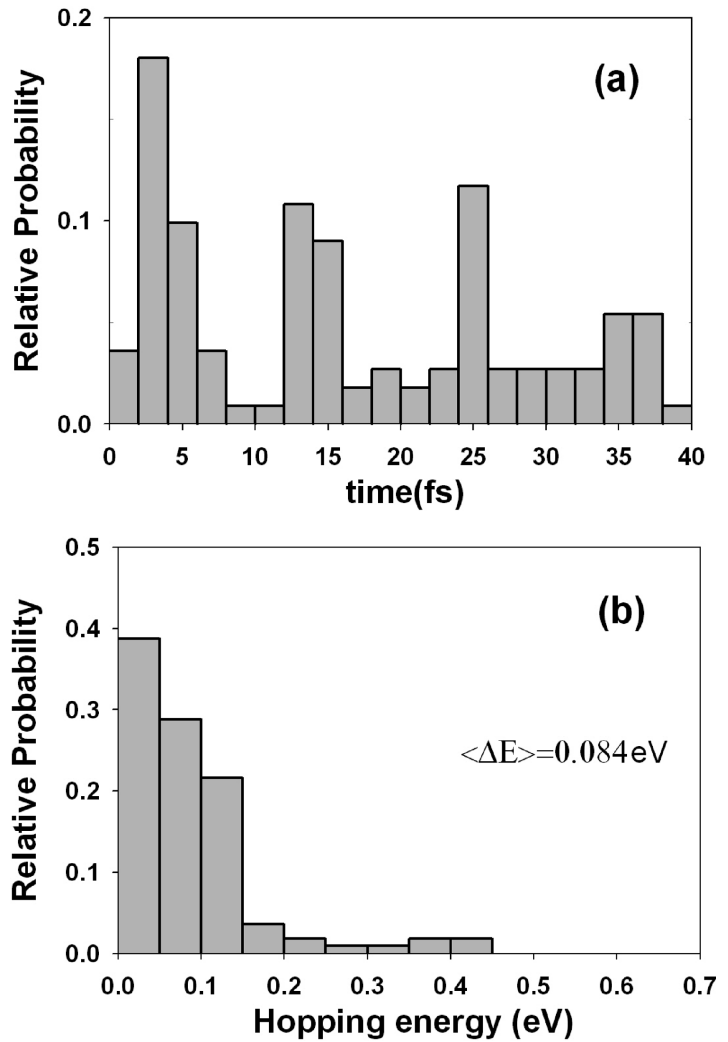

Figure 7. (a) Distribution of the number of effective $S_{2} \rightarrow S_{1}$ hops as function of time. (b) Distribution of the transition energies for the effective $S_{2} \rightarrow S_{1}$ hops.

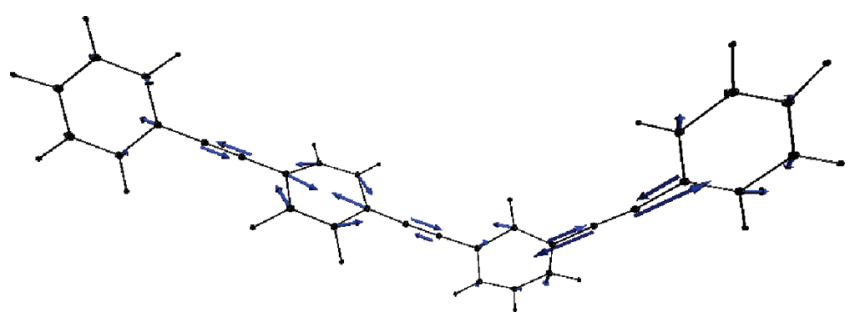

Figure 8. Nonadiabatic coupling vector $d_{1,2}$ for a typical trajectory at the time of the effective $S_{2} \rightarrow S_{1}$ hop.

coupling vector is examined. Figure 8 presents the nonadiabatic coupling vector $d_{1,2}$ for a typical trajectory at the time of the effective $S_{2} \rightarrow S_{1}$ hop. The stretching motions in the direction of the ethynylene bond, especially the one corresponding to the two-ring system, present major contributions to $d_{1,2}$. Therefore, the ethynylene stretchings should have a strong influence in the energy transfer process.

The direction of the nonadiabatic coupling vector can be interpreted as the "transition force", that is, the force on the nuclei during the hop event. ${ }^{74}$ Furthermore, the nuclear momenta are adjusted in this direction during hops. Changes in the ethynylene bond length can be expected due to the energy transfer process. Figure 9 displays the length of each of the ethynylene bonds, averaged over those trajectories with an effective $S_{2} \rightarrow S_{1}$ hop. According to previous works that localized the excitations on the different branched units, ${ }^{27,28}$ the initial optical excitation to the $S_{2}$ is localized on the two-ring system. This is in agreement with the initially larger oscillation amplitude for the triple bond of the two-ring system with respect to the triple bonds of the three-ring system shown in Figure 9. Nevertheless, the excess of vibrational energy initially detected in this bond is rapidly transferred, accompanying the electronic 

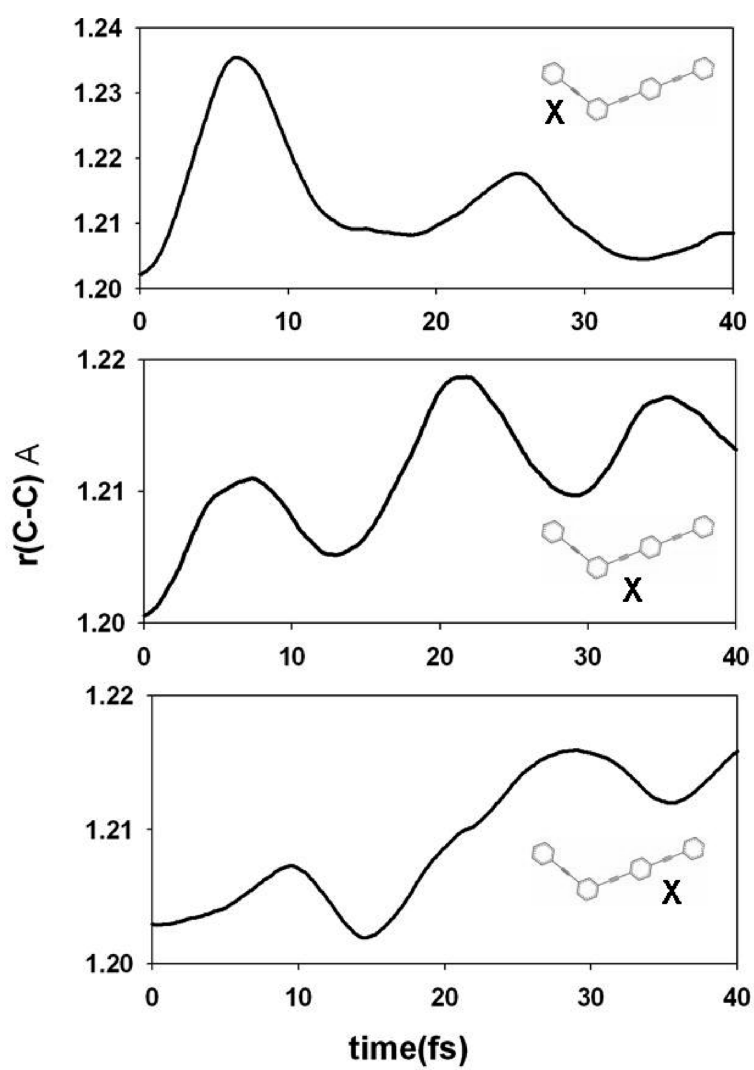

Figure 9. Average, over all the trajectories with an effective $S_{2} \rightarrow S_{1}$ hop, of the lengths of the ethynylene bonds as a function of time.
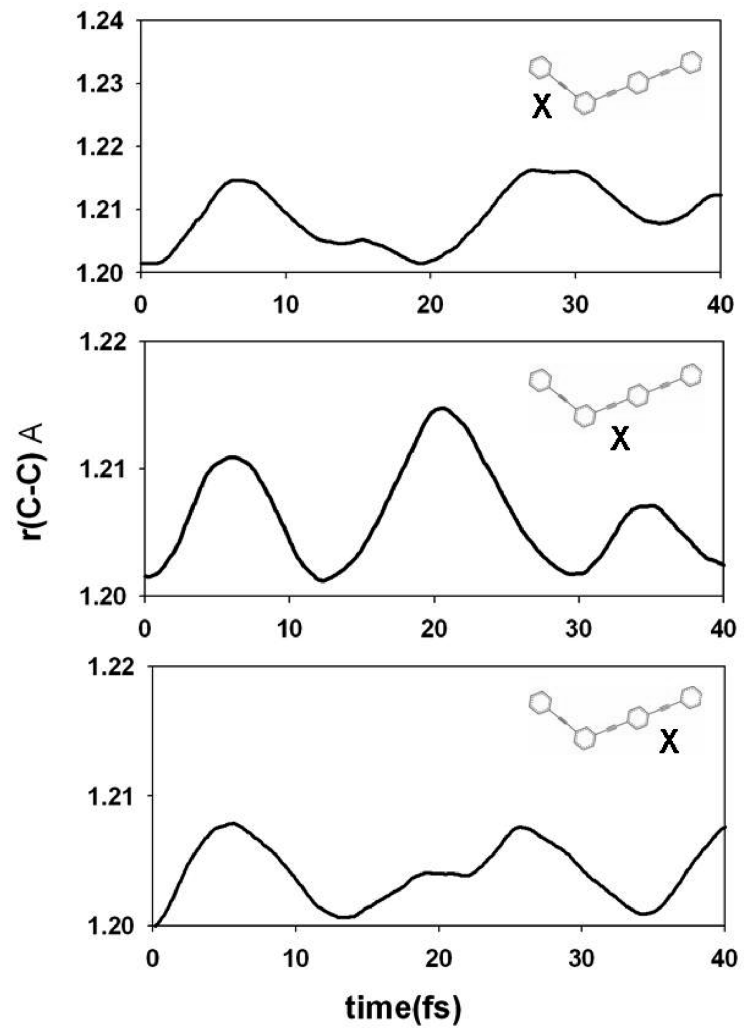

Figure 10. Average, over all the trajectories without an effective $S_{2}$ $\rightarrow \mathrm{S}_{1}$ hop, of the lengths of the ethynylene bonds as a function of time.

energy transfer $S_{2} \rightarrow S_{1}$. On the other hand, the initially not excited ethynylene bonds of the three-ring system increase their stretch lengths during the time of the simulated dynamics. This
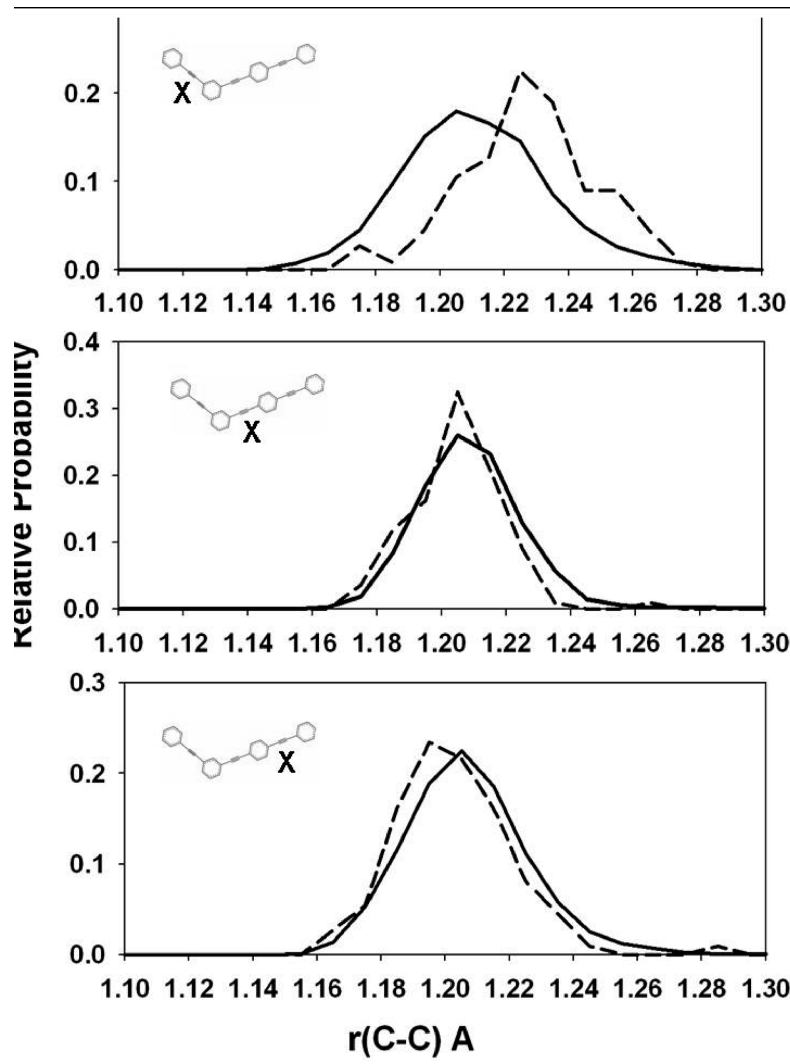

Figure 11. Histograms for lengths of the ethynylene bonds at the times of the effective $S_{2} \rightarrow S_{1}$ hops (dashed lines) and during all the molecular dynamics simulations (solid lines).

seems to indicate that an efficient unidirectional two-ring system $\rightarrow$ three-ring system vibrational energy transfer seems to follow the ultrafast electronic energy transfer.

In order to confirm that changes in the length of each of the ethynylene bonds shown in Figure 9 are due to the electronic energy transfer $S_{2} \rightarrow S_{1}$, similar analysis was performed over trajectories that do not experience an effective $S_{2} \rightarrow S_{1}$ hop. The results are shown in Figure 10. On one hand, the ethynylene bond of the two-ring system does not exhibit the large oscillation amplitude shown in Figure 9. Therefore, the large stretching of this bond seems to be a requirement for the system to reach the region close to the conical intersection. On the other hand, the ethynylene bonds of the three-ring system do not increase their lengths in time. As a result, neither the ethynylene bond of the two-ring system nor the bonds of the three-ring systems seem to change markedly their energies during the time of the dynamics.

We have analyzed the distributions of the values of the ethynylene bond lengths during hops in order to identify an internal degree of freedom with a direct and strong influence on the energy transfer process. Figure 11 displays the distributions of the values of the ethynylene bond lengths at the times of the effective $S_{2} \rightarrow S_{1}$ hops. The distributions are compared with the distributions of the corresponding values over all the trajectories. While no significant differences are observed in the values of the triple bond lengths of the three-ring system, the distribution of the bond lengths of the two-ring systems during hops is displaced to higher values. Therefore, the initial excitation of this bond observed in Figure 9 leads the system to the region of the conical intersection at the time the triple bond reaches its maximum displacement. 


\section{Conclusions}

We have performed direct nonadiabatic molecular dynamics simulations for the ultrafast process of electronic and vibrational energy transfer between meta-linked building blocks in a phenylene ethynylene dendrimer using Tully's stochastic fewestswitches algorithm. ${ }^{1,2}$ The energies, gradients, and nonadiabatic couplings have been calculated on the fly from electronic structure calculations at the CIS/AM1 level using the CEO approach.

The aim of this work was to relate the transfer of electronic state population from the $S_{2}$ to the $S_{1}$ state with an ultrafast vibrational energy transfer from the two-ring system to the threering system. For this purpose, structural and dynamical features were particularly examined in the region of strong nonadiabatic coupling.

In agreement with the near unity values of quantum yield for energy transfer observed in the nanostar, our results confirm a highly efficient electronic energy transfer between the PPE units. After the vertical photoexcitation to the $S_{2}$ state, a rapid decrease of the $S_{1}-S_{2}$ energy gap is observed, reaching a first conical intersection at $\approx 5 \mathrm{fs}$. As a consequence, the $\mathrm{S}_{2} \rightarrow \mathrm{S}_{1}$ electronic population transfer starts to take place. Thereafter, successive crossings through the region of strong couplings were reported. As a consequence, the $\mathrm{S}_{2}$ depopulates about $60 \%$ within the first $40 \mathrm{fs}$. While the nuclei motion on the $S_{2}$ pulls the system close to the conical intersection, the motion on the $\mathrm{S}_{1}$ state moves it away, enhancing the funneling signature of the energy transfer mechanism in the molecule.

The analysis of trajectories shows that the stretching motions in the direction of the ethynylene bonds induce the unidirectional energy transfer process. A large stretching of the triple bond of the two-ring system seems to be a requirement for the system to reach the region close to the conical intersection. Furthermore, changes in the stretch lengths of the ethynylene bonds indicate that an efficient unidirectional two-ring system $\rightarrow$ three-ring system vibrational energy transfer seems to follow the ultrafast electronic energy transfer.

Acknowledgment. This work was partially supported by CONICET, UNQ, the National Science Foundation grant no. CHE-0239120, and the U.S. Department of Energy and Los Alamos LDRD funds. Los Alamos National Laboratory is operated by Los Alamos National Security, LLC, for the National Nuclear Security Administration of the U.S. Department of Energy under contract DE-AC52-06NA25396. We acknowledge support of Center for Integrated Nanotechnology (CINT) and Center for Nonlinear Studies (CNLS). The authors acknowledge the University of Florida High-Performance Computing Center for providing computational resources. Computational resources were also provided by Teragrid Grant No. TG-MCA05S010. Work at University of Florida was funded by the National Science Foundation grant number CHE-0822935. AER and VDK are specially thankful to Benny for many years of incredible science and continuous support.

\section{References and Notes}

(1) Tully, J. C. J. Chem. Phys. 1990, 93, 1061. (b) Tully, J. C. Int. J. Quantum Chem. Symp. 1991, 25, 299.

(2) Hammes-Schiffer, S.; Tully, J. C. J. Chem. Phys. 1994, 101, 4657.

(3) Tomalia, D. A.; Fréchet, J. M. J. Dendrimers and Other Dendritic Polymers; John Wiley \& Sons Ltd.: West Sussex, 2001.

(4) Balzani, B.; Venturi, M.; Credi, A. Molecular Devices and Machines: A Journey into the Nanoworld; Wiley-VCH Verlag GmbH \& Co. KGaA: Weinheim, Germany, 2003.

(5) Naylor, A. M.; Goddard, W. A., III; Kiefer, G. E.; Tomalia, D. A. J. Am. Chem. Soc. 1989, 111, 2339.
(6) Bai, S. H.; Thomas, C.; Rawat, A.; Ahsan, F. Crit. Rev. Theor. Drug Carrier Syst. 2006, 23, 437.

(7) Kawa, M.; Fréchet, J. M. J. Chem. Mater. 1998, 10, 286.

(8) Mukamel, S. Nature (London) 1997, 388, 425.

(9) Kopelman, R.; Shortreed, M.; Shi, Z. Y.; Tan, W.; Xu, Z.; Moore, J. S.; Bar-Haim, A.; Klafter, J. Phys. Rev. Lett. 1997, 78, 1239.

(10) Swallen, S. F.; Kopelman, R.; Moore, J. S.; Devadoss, C. J. Mol. Struct. 1999, 485, 585.

(11) Fréchet, J. M. J. Science 1994, 263, 1710

(12) Rana, D.; Gangopadhyay, G. Chem. Phys. Lett. 2001, 334, 314.

(13) Swallen, S. F.; Zhu, Z.; Moore, J. S.; Kopelman, R. J. Phys. Chem. B 2000, 104, 3988.

(14) Inoue, K. Prog. Polym. Sci. 2000, 25, 453.

(15) Devadoss, C.; Bharathi, P.; Moore, J. S. J. Am. Chem. Soc. 1996, 118,9635 .

(16) Xu, Z.; Kahr, M.; Walker, K. L.; Wilkins, C. L.; Moore, J. S. J. Am. Chem. Soc. 1994, 116, 4537.

(17) Shortreed, M. R.; Swallen, S. F.; Shi, Z. Y.; Tan, W.; Xu, Z.; Devadoss, C; Moore, J. S.; Kopelman, R. J. Phys. Chem. B 1997, 101, 6318.

(18) Davydov, A. S. Theory of Molecular Excitons; Plenum: New York, 1971

(19) Excitons; Rashba, E. I., Sturge, M. D., Eds.; North Holland: Amsterdam, 1982. (b) Broude, V. B.; Rashba, E. I.; Sheka, E. F. Spectroscopy of Molecular Excitons; Springer: Berlin, 1985.

(20) Poliakov, E. Y.; Chernyak, V.; Tretiak, S.; Mukamel, S. J. Chem. Phys. 1999, 110, 8161.

(21) Tretiak, S.; Chernyak, V.; Mukamel, S. J. Am. Chem. Soc. 1997, $119,11408$.

(22) Mukamel, S.; Tretiak, S.; Wagersreiter, T.; Chernyak, V. Science 1997, 277, 781.

(23) Tretiak, S.; Mukamel, S. Chem. Rev. 2002, 102, 3171.

(24) Kirkwood, J. C.; Scheurer, C.; Chernyak, V.; Mukamel, S. J. Chem. Phys. 2001, 114, 2419.

(25) Tortschanoff, A.; Mukamel, S. J. Phys. Chem. B 2002, 106, 7521.

(26) Tortschanoff, A.; Piryatinski, A.; Mukamel, S. J. Lumin. 2001, 94$95,569$.

(27) Tada, T.; Nozaki, D.; Kondo, M.; Yoshizawa, K. J. Phys. Chem. B 2003, 107, 14204.

(28) Palma, J. L.; Atas, E.; Harrison, L.; Marder, T. B.; Collings, J. C.; Beeby, A.; Melinger, J. S.; Kleiman, V. D.; Krause, J. L.; Roitberg, A. E. Electronic Spectra of the Nanostar Dendrimer: Theory and Experiment. J. Am. Chem. Soc., submitted for publication.

(29) Ortiz, W.; Krueger, B. P.; Kleiman, V. D.; Krause, J. L.; Roitberg, A. E. J. Phys. Chem. B 2005, 109, 11512.

(30) Worth, G. A.; Robb, M. A.; Lasorne, B. Mol. Phys. 2008, 106, 2077

(31) Worth, G. A.; Robb, M. A. Adv. Chem. Phys. 2002, 124, 355.

(32) Ben-Nun, M.; Quenneville, J.; Martinez, T. J. J. Phys. Chem. A 2000, 104, 5161. (b) Ben-Num, M.; Martinez, T. J. Adv. Chem. Phys. 2002, $121,439$.

(33) (a) Burghardt, I.; Meyer, H. D.; Cederbaum, L. S. J. Chem. Phys. 1999, 111, 2927. (b) Worth, G. A.; Robb, M. A.; Burghardt, I. Faraday Discuss. 2004, 127, 307.

(34) Lasorne, B.; Robb, M. A.; Worth, G. A. Phys. Chem. Chem. Phys. 2007, 9, 3210

(35) Granucci, G.; Persico, M.; Toniolo, A. J. Chem. Phys. 2001, 114, 10608.

(36) Smith, B. R.; Bearpark, M. J.; Robb, M. A.; et al. Chem. Phys. Lett. 1995, 242, 27.

(37) (a) Bearpark, M. J.; Bernardi, F.; Olivucci, M.; et al. J. Am. Chem. Soc. 1996, 118, 5254. (b) Bearpark, M. J.; Bernardi, F.; Clifford, S.; et al. J. Am. Chem. Soc. 1996, 118, 169.

(38) Markwick, P. R. L.; Doltsinis, N. L. J. Chem. Phys. 2007, 126, 175102.

(39) Antol, I.; Eckert-Maksic, M.; Barbatti, M.; Lischka, H. J. Chem. Phys. 2007, 127, 234302.

(40) Zechmann, G.; Barbatti, M.; Lischka, H.; Pittner, J.; BonaèiæKoutecký, V. Chem. Phys. Lett. 2006, 418, 377.

(41) (a) Barbatti, M.; Granucci, G.; Persico, M.; Lischka, H. Chem. Phys. Lett. 2005, 410, 276. (b) Barbatti, M.; Ruckenbauer, M.; Lischka, H. J. Chem. Phys. 2005, 122, 17307.

(42) Barbatti, M.; Vazdar, M.; Aquino, A. J. A.; Eckert-Maksiæ, M.; Lischka, H. J. Chem. Phys. 2006, 125, 164323.

(43) Kleiman, V. D.; Melinger, J. S.; McMorrow, D. J. Phys. Chem. B 2001, 105, 5595.

(44) Atas, E. Ultrafast Time Resolved Excitation Dynamics in Conjugated Dendrimers. Ph.D. Thesis, University of Florida, Gainesville, FL, 2006.

(45) Hack, M. D.; Jasper, A. W.; Volobuev, Y. L.; Schwenke, D. W.; Truhlar, D. G. J. Phys. Chem. 1999, 103, 6309.

(46) Dewar, M. J. S.; Zoebisch, E. G.; Healy, E. F.; Stewart, J. J. P. J. Am. Chem. Soc. 1985, 107, 3902. 
(47) Tretiak, S.; Chernyak, V.; Mukamel, S. J. Phys. Chem. B 1998, 102,3310 .

(48) Kilina, S.; Tretiak, S. Adv. Funct. Mater. 2007, 17, 3405.

(49) Tretiak, S.; Martin, R. L.; Saxena, A.; Bishop, A. R. J. Chem. Phys. 2001, 115, 699.

(50) Tretiak, S.; Saxena, A.; Martin, R. L.; Bishop, A. R. Chem. Phys. Lett. 2000, 331, 561 .

(51) Tretiak, S.; Martin, R. L.; Saxena, A.; Bishop, A. R. Phys. Rev. Lett. 2002, 89, 97402.

(52) Franco, I.; Tretiak, S. J. Am. Chem. Soc. 2004, 126, 12130.

(53) Barbatti, M.; Ruckenbauer, M.; Lischka, H. J. Chem. Phys. 2005, $122,174307$.

(54) Hirsch, G.; Bruna, P. J.; Buenker, R. J.; Peyerimhoff, S. D. Chem.Phys. 1980, 45, 335.

(55) Cimiraglia, R.; Persico, M.; Tomasi, J. Chem. Phys. 1980, 53, 357.

(56) Martinez, T. J.; Levine, R. D. J. Phys. Chem. 1996, 105, 6334.

(57) Paterlini, M. G.; Ferguson, D. M. Chem. Phys. 1998, 236, 243.

(58) Verlet, L. Phys. Rev. 1967, 159, 98.

(59) Gear, C. W. Numerical initial value problems in ordinary differential equations; Prentice-Hall: New York, 1971.

(60) Allen, M. P.; Tildesley, D. J. Computer simulation of liquids; Oxford Science Publications: Oxford, 1987.

(61) Pastor, R. W.; Brooks, B. R.; Szabo, A. Mol. Phys. 1988, 65, 1409.
(62) Loncharich, R. J.; Brooks, B. R.; Pastor, R. W. Biopolymers 1992 , 32,523 .

(63) Eastman, P.; Doniach, S. Proteins 1998, 30, 215.

(64) Gronbech-Jensen, N.; Doniach, S. J. Comput. Chem. 1994, 15, 997.

(65) Turro, N. J. Modern Molecular Photochemistry; Benjamin/Cummings Publishing: Menlo Park, CA, 1978.

(66) Henry, B. R.; Siebrand, W. Organic Molecular Photophysics; Birks, J. B., Ed.; John Wiley \& Sons: London, 1973; Vol. 1.

(67) Desouter-Lecomte, M.; Lorquet, D. C. J. Chem. Phys. 1977, 71, 4391.

(68) Varnavski, O. P.; Ostrowsky, J. C.; Sukhomlinova, L.; Twieg, R. J.; Bazan, G. C.; Goodson, T. J. Am. Chem. Soc. 2002, 124, 1736.

(69) Lee, H.; Cheng, Y.-C.; Fleming, R. G. Science 2007, 316, 1462.

(70) Engel, G. S.; Calhoun, T. R.; Read, E. L.; Ahn, T.-K.; Manèal, T.; Cheng, Y.-C.; Blankenship, R. E.; Fleming, G. R. Nature (London) 2007, 446,782 .

(71) Bittner, E. R.; Rossky, P. J. J. Chem. Phys. 1995, 103, 8130.

(72) Bittner, E. R.; Rossky, P. J. J. Chem. Phys. 1997, 107, 8611.

(73) Schwartz, B. J.; Bittner, E. R.; Prezhdo, O. V.; Rossky, P. J. J. Chem. Phys. 1996, 104, 5942.

(74) Tully, J. C. Int. J. Quantum Chem. 2004, 40, 299.

JP900904Q 